\title{
A FOOTNOTE TO THE MULTIPLICATIVE BASIS THEOREM
}

\author{
WILLIAM GUSTAFSON
}

\begin{abstract}
We characterize those perfect fields $k$ such that for each integer $n \geq 1$, but there are but finitely many isomorphism types of $k$-algebras of dimension $n$ that are of finite representation type. Some remarks on the imperfect case are also presented.
\end{abstract}

A finite-dimensional algebra $A$ over a field $k$ is of finite representation type if it has only finitely many isomorphism types of indecomposable modules. A multiplicative basis for $A$ is a $k$-basis $B$ such that $B \cup\{0\}$ is a semigroup under the multiplication in $A$. Recently, Bautista, Gabriel, Roiter and Salmerón [1] have shown that an algebraically closed field $k$ has the property that each $k$-algebra of finite representation type has a multiplicative basis. Let us point out at once that this property characterizes algebraically closed fields. For, if a field $k$ has a finite extension field $F$, then $F$ is of finite representation type, and any multiplicative basis $B$ for $F$ would be a finite semigroup with cancellation, hence a group. Then, $F$ would be isomorphic to the group algebra $k B$, but $k B$ can never be simple if $B$ is nontrivial.

An important consequence of the theorem above is that when $k$ is algebraically closed, there are but finitely many $k$-algebras of finite representation type of any fixed $k$-dimension ("finite representation type is finite"). Let us express this by saying that $k$ has property $(\mathrm{N})$. We wish here to discuss other fields with property (N).

Recall from [3, III-29] that a field $k$ is of type $(F)$ if it is perfect and, for each $n \geq 1$, there are only finitely many $k$-isomorphism types of field extensions of degree $n$ over $k$. Examples are finite fields, local fields with finite residue field and the fields $F((T))$ of quotients of power series over algebraically closed fields $F$ of characteristic zero.

THEOREM. A perfect field $k$ has property $(\mathrm{N})$ if and only if it is of type $(F)$.

ProOF. One implication is clear. If $k$ is of type $(F)$, let $\bar{k}$ be an algebraic closure of $k$, and let $G$ be the Galois group of $\bar{k} / k$. By [2], a finite-dimensional $k$-algebra $A$ is of finite representation type if and only if $\bar{k} \otimes_{k} A$ is. Hence, it suffices to show that a $\bar{k}$-algebra $A$ of the form $\bar{k} \otimes_{k} A$ has only finitely many $k$-forms, up to isomorphism. Such forms are classified by the set $H^{1}\left(G, \operatorname{Aut}_{\bar{k} \text {-alg }}(\mathcal{A})\right)$. This set is finite by $\left[3\right.$, III-30] since $\operatorname{Aut}_{\bar{k} \text {-alg }}(\mathcal{A})$ is a linear algebraic group defined over $k$, and the proof is complete.

Let us remark on the case of imperfect fields. The degree of imperfection of a field $k$ of characteristic $p$ is that integer $r$ so that $\left[k: k^{p}\right]=p^{r}$ (or infinity, if $\left[k: k^{p}\right]$ is infinite). Thus, $k$ is perfect just when its degree of imperfection is zero. It is well

Received by the editors October 8, 1984.

1980 Mathematics Subject Classification. Primary 16A46. 
known that if $k$ has degree of imperfection two or more, then $k$ has infinitely many nonisomorphic extensions of degree $p$ (see [4, II.11.6]). Thus, an imperfect field with property $(\mathrm{N})$ must have degree of imperfection one. The best known examples of such fields are the function fields in one variable over a field of characteristic $p$. These, however, usually have many separable extensions. The most likely candidate for an imperfect field with property (N) would seem to be the separable closure of $F((T))$, with $F$ algebraically closed of finite characteristic. It should be noted that inseparable base changes generally destroy finite representation type, so descent methods are not likely to resolve the question.

Finally, it is a pleasure to acknowledge some stimulating conversations with $\mathrm{K}$. W. Roggenkamp on the subject of this note.

\section{REFERENCES}

1. R. Bautista, P. Gabriel, A. Roiter and L. Salmerón, Representation-finite algebras and multiplicative bases, preprint, Univ. Nacional Autómoma de México, 1984.

2. C. Jensen and $\mathrm{H}$. Lenzing, Homological dimensional and representation type of algebras under base field extension, Manuscripta Math. 39 (1982), 1-13.

3. J. P. Serre, Cohomologie Galoisienne, Lecture Notes in Math., vol. 5, Springer-Verlag, Berlin and New York, 1965.

4. E. Steinitz, Algebraische Theorie der Körper, J. Reine Angew. Math. 137 (1910), 167-308.

Department of Mathematics, Texas Tech University, Lubbock, TeXas 79409 